\title{
MS21-02 | Electron Configuration And Electronegativity of the Atoms Under
}

\section{COMPRESSION}

Rahm, Martin (Chalmers University of Technology, Gothenburg, SWE); Cammi, Roberto (University of Parma, Parma, ITA); Ashcroft, N. W. (Cornell, Ithaca, USA); Hoffmann, Roald (Cornell, Ithaca, USA)

The ground state configuraton of atoms is fundamental to the structure of the periodic table. Electronegativity is central for rationalizing chemical bonding, and in particular polarity and charge transfer [1]. Can the use of these atomic descriptors be extended to also predict chemistry at high pressures? To answer this question, we use a quantum mechanical model [2] to compress single atoms in a nonreactive, isotropic and neon-like environment. The effects of compression are here evaluated in a pressure range between 0 to $300 \mathrm{GPa}$ for 93 atoms. Configurational transitions, $s \rightarrow p, s \rightarrow d, s \rightarrow f$ and $d \rightarrow f$ are shown to be an essential chemical and physical consequence of compression. Our study confirms that the filling of energy levels in compressed atoms more closely follows the hydrogenic aufbau principle, where the ordering is determined by the principal quantum number. Magnetism is predicted to both increase and decrease with pressure, depending on which atom is considered. However, Hund's rule is never violated for single atoms in the considered pressure range. Drastic changes, and reordering, of electronegativity is calculated, which lead to predictions of changing chemistry and the possibly for polarity-inverted (red-ox) alloys. This extension of atomic reference data assists the working of chemical intuition at extreme pressure and can act as a guide to both experiments and computational efforts.

[1] M. Rahm, T. Zeng, R. Hoffmann, J. Am. Chem. Soc. 141 (2019) 342-351.

[2]R. Cammi, B. Chen, M. Rahm, J. Comp. Chem. 39 (2018) 2243-2250 